\title{
संगीत निर्देशक राहुल देव बर्मन का हिन्दी फ़िल्म संगीत में महत्त्वपूर्ण योगदान
}

अमृता दत्ता

पीएच.डी. शोधार्थी, संगीत एवं ललित कला संकाय, दिल्ली विश्वविद्यालय, दिल्ली

राहुल देव बर्मन जी का संक्षिप्त जीवन परिचय

श्री राहुल देव बर्मन का जन्म कोलकाता में 27 जून, 1939 को हुआ। वह कोलकाता में पले-बढ़े एवं वहीं अपनी औपचारिक शिक्षा पूरी की। आर.डी. बर्मन का घर में बोला जाने वाला नाम पंचम था। उनके पिता श्री सचिन देव बर्मन एक विख्यात संगीत निर्देशक थे, जिन्होंने हिन्दी फ़िल्म संगीत जगत को बेमिसाल गानों का खज़ाना उपहार स्वरूप दिया है। माता श्रीमती मीरादेव बर्मन संगीत की अच्छी जानकार थीं। कोलकाता में उन्हें संगीत का पहला पाठ तबले पर श्री ब्रजेन बिस्वास से मिला, (वह एक उच्चकोटि के तबला वादक थे। श्री ब्रजेन बिस्वास, श्री एस.डी. बर्मन साहब के संगीत दल में तबला वादक रहे है। वो आर.डी. बर्मन के प्रथम संगीत गुरु रहे)। तत्पश्चात् पं. सामता प्रसाद, उस्ताद अली अकबर खां, विदुषी अन्नपूर्णा देवी जैसे दिग्गजों से शिष्यत्व प्राप्त करने का सुअवसर आर.डी. बर्मन को प्राप्त हुआ।

सन् 1956 में श्री एस.डी. बर्मन द्वारा संगीतबद्ध फ़िल्म 'फंटूश' के गीत 'ऐ मेरी टोपी पलट के आ' की धुन पंचम में बनाई थी। सन् 1957 की फ़िल्म 'प्यासा' में आर.डी. बर्मन अपने पिता के सहायक रहे और एक अति लोकप्रिय गीत 'सर जो तेरा चकराये' - की धुन बनाई। उनके असाधारण संगीत प्रतिभा को देख गुरुदत्त ने अपनी एक प्रस्तावित फ़िल्म में भी आर.डी. बर्मन को संगीत निर्देशक के रूप में चुना था। (परंतु कुछ कारणवश वो फ़िल्म बन नहीं पाई) संगीत निर्देशन में आर.डी. बर्मन के प्रकृत गुरु उनके पिता ही थे। उन्होंने पंचम को संगीत की स्वरलिपि एवं गायकी के विषय में विधिवत् शिक्षा दी।

हिन्दी फ़िल्म संगीत जगत में राहुल देव बर्मन जी का आगमन 'मुमताज' के बैनर तले बनी हिन्दी फ़िल्म 'छोटे नवाब' (1961) के साथ आर.डी. बर्मन ने एक स्वतन्त्र संगीत निर्देशक के रूप में हिन्दी सिनेमा संगीत जगत में अपनी संगीत यात्रा शुरू की। इस फ़िल्म के बाद 'मुमताज' की और फ़िल्में 'भूत बंगला' (1966) एवं 'पति पत्नी' (1966) - इन दोनों फ़िल्मों के संगीत भी उन्होंने तैयार किये। फ़िल्म 'छोटे नवाब' का एक गीत 'घर आ जा घिर आये' की सफलता ने आर.डी. बर्मन को एक सफल निर्देशक के रूप में प्रतिष्ठित किया। अगले कुछ वर्षों तक उन्होंने अपने पिता के सहायक के रूप में काम किया। परंतु 'तीसरी मंजिल' (1966), पड़ोसन (1968), कटी पतंग (1970), कारवां (1971) और अमर प्रेम (1971) जैसी फ़िल्मों के लिए असाधारण गीत-संगीत देते हुए आर.डी. बर्मन लोकप्रियता के उच्च शिखर तक जा पहुँचे। आर.डी. बर्मन साहब अपने पिता की तरह एक कुशल संगीतकार थे, जिन्होंने हज़ारों-लाखों श्रोताओं के दिलों में हिन्दी फ़िल्म संगीत के माध्यम से सुरलहरी उत्पन्न की। उनके गीतों को सुनकर आज भी श्रोता मंत्रमुग्ध हो उठते हैं। उनके संगीत में भारतीय शास्त्रीय संगीत के साथ-साथ पश्चिमी संगीत की स्पष्ट झलक सुनने को मिलती हैं। लोक-संगीत का उपयोग भी उन्होंने अपने फ़िल्म संगीत में बड़ी ही कुशलता के साथ किया है। रागदारी संगीत, उपशास्त्रीय संगीत, लोकसंगीत, पाश्चात्य संगीत इन सभी संगीत धाराओं का भरपूर प्रयोग आर.डी. बर्मन साहब ने अपने गीतों में किया था। 
राहुल देव बर्मन की प्रयोगशीलता

आर.डी. बर्मन एक ऐसे दिग्गज संगीतकार थे, जिन्होंने फ़िल्मों में प्रवेश करते ही श्रोताओं की उस पुरानी परम्परा को परिवर्तित कर डाला जो एक ही प्रकार की शैली के गाने सुनना पसन्द करते थें। उन्होंने पहली बार भारतीय शास्त्रीय संगीत के चैन भरी गायन को लोक-संगीत के रंगों के साथ जोड़ा और साथ ही साथ पाश्चात्य वाद्यवृंदों के प्रयोग से एक ऐसा अनोखा एवं आकर्षक संगीत बनाया कि लोग उनकी उस अद्भुत संगीत शैली के जादू में जैसे बंध से गए थे। उनकी संगीत-धाराओं के भिन्न-भिन्न दृष्टिकोण थे - जिसके संबंध में लिखना सही माइने में आसान नहीं है। एक तो उनकी सृजनशीलता का विस्तार लगभग 1500 गीतों के साथ-साथ 292 हिन्दी फ़िल्में, 31 बंगाली फ़िल्में, 8 अन्य भाषाओं में बनी फ़िल्में; 'पन्टेरा' और 'दिल पड़ौसी' है जैसे प्राइवेट एलबम तक विस्तृत हैं। दूसरी खास बात यह है कि उनकी बनाई हुई धुनों में विविधता होती है। एक ओर परम्परागत विशुद्ध भारतीय गीत-संगीत और दूसरी ओर विश्व संगीत की विभिन्न सांगीतिक परम्पराओं से प्रभावित संगीत और अभ्यस्त कानों को विजातीय, अगम्य और कभी-कभी कानों को अप्रिय लगता संगीत - इन सभी के प्रयोगों के कारण उनका संगीत वैचित्रतापूर्ण होता था। तीसरी ख़ास बात थी।

उनकी प्रयोगशीलता, लय-ताल-छन्द के साथ की नई-नई कोशिशों, कभी-कभी असंगतिपूर्ण ध्वनियों का समावेश, स्केल परिवर्तन, पौलिफौनी और हॉरमनी जैसी विदेशी विधियों का उपयोग इत्यादि। इन सभी प्रयोगों के कारण आर.डी. बर्मन के गीतों के श्रोता भी दो विपरीत दलों में बँट गये हैं। एक पक्ष में वो लोग हैं जो परम्परावादी हैं एवं जिन्हें पंचम का नया प्रयोगवादी संगीत शोर-शराबे से भरा संगीत लगता है। दूसरी तरफ असंख्य युवा प्रेमी भी हैं जिन्हें पंचम की संगीत शैली ने मुग्ध कर दिया था। पंचम को उनकी समग्रता के साथ समझने के लिए यह कहना जरूरी होगा कि पारम्परिक संगीत रचने के अलावा पंचम का मुख्य महत्त्व यह था कि वे एक क्रांतिकाल में एक नए युग के लिए नई संगीत रचना की प्रक्रिया को लेकर फ़िल्म संगीत जगत में उभरे एवं श्रोताओं को मंत्रमुग्ध किया। उनके गीतों में नवीन प्रवृत्तियों के साथ परम्परा का पक्ष भी बहुत सबल था। हिन्दी फ़िल्मी संगीत जगत में नवीन प्रवृत्ति की शुरुआत 1961 के आस-पास शंकर-जयकिशन के संगीत में देखी जा सकती है। सन् 1963 के आस-पास लक्ष्मीकांत-प्यारेलाल भी अपनी स्वतंत्र संगीत रचना के साथ सामने आए। लेकिन नवीनता का सबसे दूरगामी, सबसे ज़्यादा क्रांतिकारी उद्घोष राहुल देव बर्मन द्वारा 'तीसरी मंजिल' (1966) के लिए बनाए गये संगीत के रूप में सामने आया। संगीतकार के रूप में उनकी वास्तविक पहचान नासिर हुसैन की इसी फ़िल्म से हुई थी।

इस फ़िल्म में उन्होंने जैज़ संगीत के साथ भारतीय शास्त्रीय संगीत का इतना सुंदर तालमेल बिठाया, जो अत्यन्त प्रशंसनीय था। इनके संगीत की यह विशेषता थी कि पाश्चात्य संगीत का प्रयोग करते हुए भी उन्होंने शास्त्रीय संगीत की सुंदरता बरकरार रखी। इस तरह के प्रयोग से संगीत की जो नई शैली उन्होंने बनायी वह पंचम शैली के नाम से विख्यात हुई। इसी फ़िल्म का लोकप्रिय गीत 'आजा-आजा मैं हूँ प्यार तेरा', 'अल्ला अल्ला इंकार तेरा' एवं 'इसके बाद आ-आ-आजा, आ-आ आजा' - गीत ने मानों संगीत की दुनिया में तहलका मचा दिया। इसी फ़िल्म का और एक गीत 'ओ मेरे सोना रे सोना रे सोना रे', 'दे दूँगी जान जुदा मत होना रे' - में 
पाश्चात्य, लोकसंगीत और शास्त्रीय संगीत के विविध रंगों की मनमोहक मिलावट के साथ एक और खास बात भी थी वह है 'शब्दों से ज़्यादा स्वरों का महत्त्व'।

उनके द्वारा बनाई धुनों में एक सुरीला चमत्कार था जो गायकों के स्वरों को भी साजों की तरह बजा सकता था। राहुल की इसी नवीन संगीतधारा ने उन्हें 'युवा पीढ़ी' का पसंदीदा संगीतकार बना दिया था एवं साथ ही साथ श्रोताओं के उस वर्ग ने भी उन्हें अपना लिया जो गज़ल के नाम पर मदन मोहन, लोक संगीत के नाम पर एस.डी. बर्मन, नौशाद; पाश्चात्य संगीत के नाम पर सी. रामचंद्र और आर्केस्ट्रा के नाम पर केवल मात्र शंकर-जयकिशन को ही सुनना पसंद करते थे। 'तीसरी मंजिल' की सफलता के बाद न जाने कितनी मंजिलें पार करते हुए राहुल देव बर्मन लोकप्रियता के उच्च शिखर पर पहुँच गए। विख्यात गीतकार जावेद अख़्तर जी ने कहा कि "पंचम के आने से पहले हिन्दी फ़िल्म संगीत कुछ क्लीशे (दोहराव भरा) होने लगा था। जबकि समाज बदल रहा था, मूल्य बदल रहे थे, (जीवन की) रफ्तार बदल रही थी। आर.डी. बर्मन अपने दौर का समसामयिक इंसान था और समाज में जो नये प्रभाव आये थे वे हमें उनके संगीत में भी दिखाई देते हैं। जाहिर है, हमें पंचम के संगीत को उस दौर के वृहत्त सामाजिक, सांस्कृतिक परिप्रेक्ष्य में और उसके अन्तर्गत खुद फ़िल्मों में आ रहे बदलावों के संदर्भ में देखना होगा। ${ }^{\prime \prime 1}$

राहुल देव बर्मन - एक क्रांतिकारी संगीतकार

राहुल देव बर्मन एक क्रांतिकारी संगीतकार थे। उन्होंने आधुनिक वाद्ययन्त्रों में लोकसंगीत का समन्वय कर फ़िल्म संगीत में अभिन्न प्रयोग किये। उनके इन सब प्रयोगों के कारण उनका विरोध भी हुआ। उनके ऊपर यह आरोप भी लगाया गया कि वह अपने इन आधुनिक प्रयोगों के माध्यम से भारतीय शास्त्रीय संगीत को विकृत कर रहे हैं। हिन्दी फ़िल्मी गीतों में मैलोडी के स्थान पर वाद्य-यन्त्रों का शोर गुंजा देने का आरोप उन पर लगाया गया था। परंतु अपने ऊपर लगाए गए ऐसे आरोपों के कारण राहुल देव बर्मन जी कभी भी विचलित नहीं हुए। करीब साढ़े तीन सौ गीतों में अपना आधुनिक संगीत देकर उन्होंने फ़िल्म संगीत में एक अच्छी पहचान बनायी, जिस पहचान ने उन्हें संगीतकार के रूप में उच्च शिखर तक पहुँचाया। उनके द्वारा किये गये नये परिवर्तनों के बारे में उनका मानना था - "जब बाबा ने 'बाजी' का संगीत तैयार किया था तब उन पर भी भारतीय संगीत को विकृत करने का आरोप लगा था। लोग परिवर्तन का हमेशा विरोध करते हैं लेकिन परिवर्तन तब भी होता है। उसे कोई नहीं रोक सकता। पाश्चात्य संगीत को हमारे यहाँ आना ही था। यह मेरे जरिए आता या किसी और के माध्यम से आता पर आता तो जरूर। हमारे रहन-सहन में जब पश्चिमी असर हावी हो गया है तो संगीत कैसे अछूता रह पाता? समय बदलने के साथ-साथ सब कुछ बदल जाता है और मैंने फ़िल्म संगीत को बदले हुए समय के मुताबिक बदला ।"2

हिन्दी फ़िल्मों को एक नया एवं धड़कता हुआ संगीत देने में राहुल देव बर्मन का नाम विशेष उल्लेखनीय था। उन्होंने हिन्दी गीतों में जैज़ एवं पॉप संगीत का रंग भरा और साथ ही साथ संगीत के मदभरे दौड़ की भूमिका भी उन्होंने लिखी। संगीतकार आर.डी. बर्मन ने हिन्दी फ़िल्मों गीतों में जो अनूठा प्रयोग किया था वो बेमिसाल है, कुछ उदाहरण इस प्रकार दिए जा सकते हैं -

1 स्वर सरिता (7वां वर्ष विशेष), जुलाई 2014, 'हिन्दी फ़िल्म संगीत', पृ. 36.

2 जनसत्ता, दिल्ली, 5 जनवरी, 1994, "फ़िल्म संगीत को मस्ती के कई रंग दिए पंचम ने'।

Swar Sindhu : A National Peer-Reviewed Journal of Music | UGC approved 63647 
फ़िल्म 'पड़ोसन' के गीत 'मेरे सामने वाली खिड़की में' राहुल देव बर्मन जी ने एक सींक वाली झाडू को एक वाद्ययंत्र के रूप में इस्तेमाल किया था। इस गीत के प्रारंभ में केष्टो मुखर्जी झाडू बजाकर गीत की शुरुआत करते हुए दिखाई पड़ते हैं। यह गीत को उनके प्रयोगवादी संगीत का एक सुंदर उदाहरण कहा जा सकता है। गुलज़ार की फ़िल्म 'परिचय' में नायक जितेन्द्र जब तांगे पर बैठकर "मुसाफिर हूँ यारों, ना घर हैं ना ठिकाना' - गीत को गाते है तब घोड़ों की टापों और तांगे चलाने की आवाज़ उन्होंने तबले से निकलवाई थी, इसी प्रकार गुलज़ार की और एक फ़िल्म 'किताब' के गीत 'मास्टर जी की चिट्ठी आई' - में लकड़ी के फट्टे से लय निकाली गई थी। यह सब राहुल देव बर्मन जी के असाधारण आर्केस्ट्राइजेशन का ही कमाल था। आर्केस्ट्राइजेशन की तरह 'रिदम कंटीन्यूटी' अर्थात् लय-निरंतरता में थी आर.डी. बर्मन ने कुछ नए प्रयोग किए। इस बारे में दो उदाहरण दिए जा सकते हैं। एक तो गुलज़ार की फ़िल्म 'खुशबू' के गीत 'ओ माझी रे' और दूसरी विधु विनोद चोपड़ा की फ़िल्म '1942-ए लव स्टोरी' के गीत 'एक लड़की को देखा तो ऐसा लगा' - इन दोनों गीतों में रिदम कंटीन्यूटी की खूबसूरती सुनने को मिलती है।

राहुल देव बर्मन द्वारा हिन्दी फ़िल्म संगीत में किए गए नए प्रयोग

संगीतकार राहुल देव बर्मन हिन्दी फ़िल्म गीतों में नए प्रयोग करने में अत्यन्त पारदर्शी थे। उनके पिता संगीतकार सचिव देन बर्मन जी बंगाल और पूर्वोत्तर प्रदेशों की लोकधुनों का अधिकाधिक प्रयोग अपने फ़िल्मी गीतों में करते थे। पिता से ही उन्हें लोक संगीत एवं शास्त्रीय संगीत विरासत में मिला था। साथ ही साथ वो पॉप और जैज़ संगीत पसन्द करते थे एवं हिन्दुस्तानी संगीत के साथ इन सभी संगीत का बेहतरीन प्रयोग करते थे। राहुल देव बर्मन पॉप संगीत में बीटल, एलविस प्रेसले को बहुत सुनते थे। फिल कौलिंस को वह खास तौर पर पसंद करते थे। जैज़ संगीतधारा में किन्सी जोंस और काउंट बासले को वे सुनते हैं। इसके साथ-साथ हिन्दुस्तानी शास्त्रीय संगीत में उनकी जो विधिवत् तालीम थी, उसके विषय में पहले ही बताया गया है। शास्त्रीय संगीत जगत के दिग्गज कलाकार जैसे उस्ताद अली अकबर खाँ साहब एवं पंडित सामता प्रसाद जी के सान्निध्य में उनकी शास्त्रीय संगीत की शिक्षा प्राप्त हुई थी। इन सभी संगीत धाराओं को आधार बनाकर ही वे फ़िल्मों के लिए खूबसूरत गीत रचने की स्थिति में आ पाए। एक ऊँचे दर्जे के संगीतकार होने के साथ-साथ उनमें और एक खूबी भी थी। राहुल देव बर्मन साहब सुंदर माउथ ऑर्गन बजाते थे।

'दोस्ती' फ़िल्म में उन्होंने लक्ष्मीकांत प्यारेलाल के लिए माउथ ऑर्गन बजाया। शंकर जयकिशन, हेमन्त कुमार और सलिल चौधरी जैसे संगीतकारों ने भी अपनी फ़िल्मों में उनसे माउथ ऑर्गन बजवाया। अपने पिता के सहायक के रूप में फ़िल्म 'आराधना' में भी 'मेरे सपनों की रानी गीत' में उन्होंने माउथ ऑर्गन बजाया। वो हारमोनियम एवं कीबोर्ड भी अच्छा बजा लेते थे। कई सारे वाद्ययन्त्रों पर महारत हासिल करने के लिए राहुल देव बर्मन 'प्यासा' से लेकर 'गाइड' फ़िल्म तक अपने पिता के सहायक रहे। 'गाइड' फ़िल्म का गीत 'दिल ढल जाए हाय रात न जाए' को बनाते समय सचिन देव बर्मन को दिल का दौरा पड़ा था। इस गीत का मुखड़ा एवं एक अंतरा तैयार था। इस गीत की संगीत रचना को राहुल देव बर्मन जी ने पूरा किया था।

राहुल देव बर्मन द्वारा गाए कुछ गीत

राहुल देव बर्मन जी ने अपने अनोखे अन्दाज़ में कुछ गीत भी गाए। 'भूत बंगला' फ़िल्म में 'मैं भूखा हूँ, तुझे खाऊंगा'; 'शोले' में 'महबूबा-महबूबा'; 'शान' में 'यम्मा यम्मा यम्मा यम्मा ये खूबसूरत समा' 
और 'कारवाँ' में उनके अनोखे अन्दाज़ में गाया गीत 'मोनिका, ओ माई डार्लिंग' आज भी श्रोताओं को मन्त्रमुग्ध कर देती है।

1966 में नासिर हुसैन की फ़िल्म 'तीसरी मंजिल' हिन्दी फ़िल्म संगीत में एक क्रांति ले आई। इस क्रान्ति के जन्मदाता थे राहुल। उन्होंने इस फ़िल्म में पूरी तरह से तेज और पश्चिमी संगीत का प्रयोग किया। इस फ़िल्म के सभी गीत 'ओ हसीना जुल्फों वाली', 'ओ मेरे सोना रे सोना रे सोना रे', 'आजा आजा मैं हूँ प्यार तेरा' और 'दीवाना मुझसा नहीं इस अंबर के नीचे' - अपनी विशिष्ट धुनों की वजह से विलक्षण रहे।

अपनी फ़िल्मों में एक अलग संगीत देने का जो सिलसिला राहुल देव बर्मन जी ने शुरू किया था या फिर यह कह सकते है कि उन्होंने फ़िल्मी गीतों की जो दिशा बदली उसका मूल कारण यह था कि फ़िल्म संगीत के मिजाज में बहुत कुछ नया करने की गुंजाइश बाकी थी। पश्चिमी संगीत का असर महसूस किया जाने लगा था। राहुल देव बर्मन को यह महसूस हुआ था कि लोगों की रूचि तेजी से बदल रही है। रॉक एंड रोल का हिन्दी फ़िल्म संगीत पर प्रभाव पड़ने लगा था। 'भूत बंगला' में राहुल देव बर्मन ने 'आओ ट्विस्ट करे' से 'ट्विस्ट नृत्य' को भारतीय फ़िल्मों में प्रवेश करवाया। यह एक नया प्रयोग था। परंतु यह भी एक सोचने की बात है कि इसी फ़िल्म में राहुल देव बर्मन ने 'जागो सोने वालो, सुनो मेरी कहानी' (किशोर कुमार) जैसे जीवनमुखी गीत की भी रचना की। 'तीसरा कौन' फ़िल्म का गीत 'प्यार का फसाना बना ले दिल दीवाना' - संगीत में उनकी गहरी सोच को साबित करता है।

'तीसरी मंजिल' के सहारे पंचम दा ने हिन्दी फ़िल्म संगीत जगत के प्रेमियों को यह सिखाया था कि सितार, तबला, ढोलक, सारंगी आदि भारतीय वाद्य यन्त्र के साथ-साथ यदि बिजली द्वारा चालित वाद्ययन्त्रों का भी इस्तेमाल किया जाए तो हिन्दी फ़िल्मी गीतों में नयापन आएगा। जब राहुल देव बर्मन फ़िल्म संगीत जगत में आए तब तक फ़िल्मों में 'शोला जो भड़के जैसे गीतों की रचना हो चुकी थी। पर हिन्दी फ़िल्मी गीतों में ड्रम सैट, कंगो, कीबोर्ड, गिटार आदि वाद्ययन्त्रों का सही इस्तेमाल राहुल देव बर्मन जी ने सूझबूझ के साथ किया था। इन सभी वाद्ययन्त्रों के प्रयोगों से सफल हुए गीतों को आज भी लोकप्रिय माना जाता है।

'तीसरी मंजिल' के बाद नासिर हुसैन की एक फ़िल्म 'बहारों के सपने' में भी राहुल देव बर्मन जी ने संगीत दिया। इस फ़िल्म का एक खूबसूरत गीत था 'आजा पिया तोहें प्यार दू' इस गीत में कहीं भी पश्चिमी संगीत का असर दिखाई नहीं दिया। सातवें दशक में बनी तीन फ़िल्में हैं - 'हरे राम हरे कृष्ण', 'अमर प्रेम' और 'कटी पतंग'। इन तीनों फ़िल्मों के गीतों को सुनकर राहुल देव बर्मन जी की विविधता का अंदाजा लगाया जा सकता है। 'हरे राम हरे कृष्ण' फ़िल्म का गीत 'दम मारो दम मिट जाए गम' ने तो हंगामा मचा दिया था। इसी फ़िल्म के दो अन्य गीत 'कांछी रे कांछी रे प्रीत मेरी सांची' और 'फूलों का तारों का सबका कहना है', 'दम मारों दम' से एकदम अलग थे। सन् '1970' में बनी फ़िल्म 'कटी पतंग' का गीत 'मेरा नाम है शबनम, प्यार से लोग मुझे शब्बो कहते हैं' - इस गीत में राहुल देव बर्मन ने एक नया प्रयोग किया था। इस फ़िल्म के बाकी गीतों में - 'ये शाम मस्तानी', 'प्यार दीवाना होता है मस्ताना होता है', 'ये जो मोहब्बत है ये उनका है काम', 'जिस गली में तेरा घर न हो बालमा', 'ना कोई उमंग है', 'आज ना छोड़ेंगे' - इन सभी गीतों में राहुल देव बर्मन जी ने नए-नए अन्दाज दिए। 
सन् '1971' में बनी फ़िल्म 'अमर प्रेम' के गीतों को सुनकर बिल्कुल अलग ढंग की संगीत रचना का आनन्द मिलता है। इस फ़िल्म के गीत 'चिंगारी कोई भड़के तो सावन उसे बुझाए', 'रैना बीती जाए, श्याम ना आए', 'कुछ तो लोग कहेंगे लोगों का काम है कहना' और 'ये क्या हुआ कैसे हुआ कब हुआ' जैसे गीतों ने यह साबित कर दिया था कि केवल शोरगुल वाले तेज संगीत ही नहीं, अपितु राहुल देव बर्मन जी के गीतों में सुमधुर संगीत से भरी मीठी धुन भी सुनने को मिलती है।

विख्यात, लेखक-निर्देशक गुलज़ार की कई फ़िल्मों में पंचम ने यादगार संगीत दिया। सन् '1972' में बनी फ़िल्म 'परिचय' में सबसे मधुर गीत था 'बीती ना बिताई रैना' जिसकी धुन पंचम ने एक होटल के कमरे में बैठकर बनायी थी। इस गीत को गाने के लिए गायिका लता को राष्ट्रीय पुरस्कार मिला था। 'परिचय' फ़िल्म के बाद गुलज़ार की अधिकांश फ़िल्मों में राहुल देव बर्मन जी ने ही संगीत दिया। गुलज़ार एवं राहुल देव बर्मन का अद्भुत तालमेल हमें 'आँधी' (1975) फ़िल्म के गीतों में सुनने को मिलता है। इस फ़िल्म के तीनों गीत 'तेरे बिना जिंदगी से कोई शिकवा तो नहीं', 'किस मोड़ से जाते हैं' एवं 'तुम आ गए हो नूर आ गया है' - हिन्दी फ़िल्म संगीत जगत के लिए अविस्मरणीय गीत बन गए थें।

गुलज़ार द्वारा निर्देशित और एक फ़िल्म है 'किनारा' (1977)। इस फ़िल्म के अधिकतर गीत शास्त्रीय संगीत के आधार पर बनी हुई है। इस फ़िल्म के गीतों को सुनकर भी यह अन्दाज़ा लगाया जा सकता है कि राहुल देव बर्मन एक गुणवान संगीतकार है। गुलज़ार की मुक्त छंद वाली कविता की संगीत रचना थी राहुल देव बर्मन अत्यन्त कुशलता के साथ करते थे।

1987 में गुलज़ार और राहुल देव बर्मन जी ने मिलकर एक गैर फ़िल्मी एलबम बनाया था जिसका नाम था 'दिल पड़ोसी है'। इस एलबम में पश्चिमी संगीत धारा जैज़, रवीन्द्र संगीत तथा भारतीय हिन्दुस्तानी शास्त्रीय संगीत का सुंदर समन्वय हमको सुनने को मिलता है। सन् 1988 में बनी 'इजाज़त' मानवीय भावनाओं का जिन्दगी पर जो गहरा प्रभाव होता है, उसी का एक सुंदर चित्रण है। यह एक भावनात्मक फ़िल्म है और इसके गीत गुलज़ार साहब ने लिखे हैं। उन गीतों को स्वरबद्ध किया था राहुल देव बर्मन जी ने। मुक्त छंद में बनी गीत का एक सुंदर उदाहरण है इसी फ़िल्म का गीत 'मेरा कुछ सामान तुम्हारे पास पड़ा है'। इस भावुक गीत को आशा जी ने गाया ओर उन्हें इस गीत के लिए राष्ट्रीय पुरस्कार से सम्मानित भी किया गया था। इस फ़िल्म के बाकी गीत 'छोटी सी कहानी से', 'कतरा कतरा', 'खाली हाथ श्याम आए है' भी राहुल देव बर्मन जी की असाधारण संगीत प्रतिभा का निर्देशन हैं।

राहुल देव बर्मन की कार्य पद्धति

पंचम ने आर्केस्ट्रा का प्रयोग बड़ी खूबसूरती के साथ फ़िल्मों में किया था। कुछ फ़िल्मों में आर्केस्ट्रा का प्रयोग करके उन्होंने अति सुंदर प्रभाव उत्पन्न किया है। कुछ उदाहरण हैं - फ़िल्म कारवाँ (1971) का गीत 'पिया तू अब तो आजा', फ़िल्म 'हम किसी से कम नहीं' (1977) का गीत 'बचना ए हसीनों लो मैं आ गया' इत्यादि उल्लेखनीय हैं। इन दोनों फ़िल्मों के बाकी गीत भी अति लोकप्रिय साबित हुए।

हिन्दी फ़िल्मी संगीत में उन्होंने अनेकों परिवर्तन किए। विभिन्न भारतीय एवं पाश्चात्य वाद्ययन्त्रों के प्रयोगों से अनेक लोकप्रिय गीतों का निर्माण किया। जाने-माने गीतकार श्री गुलजार साहब के 
अनुसार राहुल देव बर्मन जी 'मेलडी' ज़्यादा पसंद करते थे। अपने गीतों में मधुरता उत्पन्न करने के लिए वो पाश्चात्य तरीके का प्रयोग करते थे। वो अपने गीतों में गिटार का प्रयोग ज़्यादा करते थे और साथ में कीबोर्ड, अक्टोपेड का प्रयोग भी करते थे। पंचम दोनों पद्धतियों में धुन बनाते थे। पहले धुन बनाकर बाद में शब्द भरना एवं पहले शब्द को चुनकर बाद में उसमें समुचित धुन देना - दोनों तरीकों से वो अपने गीतों को स्वरबद्ध करते थे। पंचम की दृष्टि से गीतों के लिए धुन बनाना एक रचनात्मक कला होती है।

पंचम आर्केस्ट्रा और इलैक्ट्रॉनिक वाद्ययन्त्र के साथ जैसे पाश्चात्यीकृत संगीत की रचना करते थे उसी तरह शांत, मधुर, मैलोडी भरी गीतों को भी वो उसी अन्दाज़ से रचने की कोशिश करते थे। परंतु कुछ-कुछ फ़िल्मों में उनके संगीत में इलैक्ट्रॉनिक्स हावी हो गया। सन् 1982 में बनी फ़िल्म 'सनम तेरी कसम' फ़िल्म का गीत कुछ इस प्रकार का ही था।

राहुल देव बर्मन जी का मानना था, 'पहले जमाने में गीत, शब्दों पर ज़्यादा ज़ोर रहता था, अब फ़िल्म निर्देशक शॉट डिवीजन, फ़िल्मकरण पर ज़्यादा बात करते हैं। पहले संगीत छोटा (सीमित) हुआ करता था - इंटर्ल्यूड, इंट्रोडक्शन, कोडा, क्रैसेंडो म्यूजिक - ये बस कम होते थे, लेकिन अब ये स्टाइल चल पड़ा है। हर गाने में म्यूजिक का प्रभाव ज़्यादा हो गया, साथ ही द्विअर्थी गाने भी आ गये।"1

पंचम के घनिष्ठ मित्र और अपनी 'जवानी दीवानी' 1972 से लेकर 'इंद्रजीत' 19 तक निरन्तर 12 फ़िल्मों में पंचम का संगीत लेने वाले निर्देशक रमेश बहल द्वारा 1989 में व्यक्त किये गये इन विचारों को इसी संदर्भ में समझना जरूरी है; पंचम ने अनेक बार अमेरिका भ्रमण किया। उन्हें वहाँ की पाश्चात्य संगीत व्यवस्था भा गई। उन्होंने वहाँ 27 ट्रैक की रिकॉर्डिंग देखी और भारत के 6 ट्रैक पर उन्हें अफ़सोस हुआ। इलैक्ट्रॉनिक वाद्ययंत्रों ने उन्हें अचंभित कर दिया।

'“'ीमा' (1984) के 'मानूंगी ना तेरी मनमानी मैं तो मानूंगी ना रसिया' में स्वदेशी भावभूमि और पाश्चात्य आर्केस्ट्रेशन का मेल खटकता है। इसी तरह 1986 के 'काबुल से आया हैं मेरा दिलदार' में थीम मध्यपूर्वी है लेकिन आर्केस्ट्रेशन वहाँ टकसाली, आधुनिक और पाश्चात्य टाइप का है। 'आमने सामने' 1982 में मामूली धुनों को आर्केस्ट्रेशन के सहारे पार लगाने का प्रयास है। 'बेलगाम' 1987 में मामूली गीतों को विस्तृत 'प्रिल्यूड्स' (शुरुआती वाद्य संगीत) का सहारा दिया गया है। 'महान' 1983 का 'प्यार में दिल पे मार के गोली' में तुकबंदी को गोलियों की आवाज़ और मुफ्त के हल्ले-गुल्ले से सजाया गया है। 'गुरुदेव' 1993 का 'आजा सुनले सदा' गीत छोड़कर साज-गिरी के साथ खिलवाड़ में पड़ जाने का उदाहरण पेश करता है जबकि 'आंचल' (1980) के 'बस मेरी जां बस मानले मेरी बात' में गीत और धुन के बीच सामंजस्य का अभाव देखा जा सकता है।'2

इन सभी विवादों के बावजूद भी इस बात को मानने से कोई भी इंकार नहीं कर सकता कि उन्हें नई-नई धुनें बनाने का शौक बहुत रहा है। एक तरफ जहाँ उन्होंने किशोर कुमार जी से 'मेरी प्यारी बिन्दू', 'एक चतुर नार' (पड़ोसन 1968) जैसे हास्य गीतों को गवाया। वहीं उन्होंने महबूबा (1976) फ़िल्म में 'मेरे नैना सावन भादो' जैसा दर्द भरा रागाधरित गीत भी किशोर जी से गवाया है।

1 हिन्दी फ़िल्म संगीत - विजय वर्मा, 34 प्रकाशन, तिथि 1 सितंबर 2014, स्वर सरिता, पृ. 36. 2 वही, पृ. 36.

Swar Sindhu : A National Peer-Reviewed Journal of Music | UGC approved 63647 
(राग-शिवरंजनी पर आधारित)। उनके संगीत निर्देशन में बनी मेलोडी-प्रधान प्रेम-गीत तो अनगिनत ही हैं जो आज भी लोग गाते-गुनगुनाते हुए नहीं थकते।

साथ-ही-साथ शास्त्रीय संगीत के विभिन्न रागों पर आधारित उनकी कुछ प्रमुख रचनाएँ हैं फ़िल्म 'बुड्ढा मिल गया' (1971) का गीत 'आयो कहाँ से घनश्याम', गायक/गायिका-मन्ना डे एवं अर्चना, राग-खमाज, ताल-अद्धा + तीनताल + कहरवा। फ़िल्म 'अमरप्रेम' (1971) का गीत 'रैना बीती जाए', गायिका-लता, राग-गुर्जरी तोड़ी + तोड़ी + खमाज/ललित; ताल-कहरवा। फ़िल्म 'परिचय' (1972) का गीत 'मितवा बोले मीठे बैन' गायक-भूपिन्दर, राग-भिन्न षड्ज + माँझ खमाज + रागेश्री + मारुबिहाग + पुरियाधनश्री; ताल-अच्छा तीन ताल। 'बीती ना बिताई रैना विरहा की मारी रैना' गायक/गायिका-लता एवं भूपिन्दर, राग-बिहाग + यमन + कलावती; ताल-कहरवा। फ़िल्म 'आँधी' (1975) का गीत 'तेरे बिना जिंदगी से कोई शिकवा तो नहीं' गायक/गायिका-किशोर कुमार एवं लता, राग-किरवानी + नटभैरवी + काफ़ी, ताल-कहरवा। 'तुम आ गए हो नूर आ गया है' गायक / गायिका-किशोर कुमार एवं लता, राग-नटभैरवी, ताल-कहरवा। फ़िल्म 'किनारा' (1977) का गीत 'नाम गुम जाएगा चेहरा ये बदल जाएगा' गायक/गायिका-लता एवं भूपिन्दर, राग-यमन + खमाज, ताल-कहरवा। 'अब के ना सावन बरसे' गायिका-लता, राग-झिंझोटी + खमाज, ताल-कहरवा। 'गुरु वंदना' गायक-पंकज मित्रा, राग-तोड़ी, पारम्परिक रचना, तालविहीन। 'तराना' गायक-गिरीश वझलवार, राग-हमीर, पारम्परिक रचना, ताल-एकताल, 'मीठे बोल बोले' गायक / गायिका-लता एवं भूपिन्दर, राग-गोपिका बसन्त + भैरवी + पटद्वीप + चन्द्रकौंस, ताल-अद्धा तीनताल + तीनताल + चारताल + कहरवा। 'कोई नहीं है कहीं' गायक-भूपिन्दर सिंह, राग-तोड़ी + किरवानी + पहाड़ी, ताल-दादरा। फ़िल्म 'कुदरत' (1981) का गीत 'हमें तुमसे प्यार कितना यह हम नहीं जानते', गायक/गायिका-किशोर कुमार, परवीन सुल्ताना, आशा भोंसलें, राग-नटभैरवी + किरवानी, ताल-कहरवा। इन सभी गीतों को सुनने के बाद इस सच्चाई को नकारा नहीं जा सकता है कि उन्हें शास्त्रीय संगीत का भी भरपूर ज्ञान है और शास्त्रीय संगीत के रागों के आधार पर भी राहुल देव बर्मन सुमधुर धुनें बना सकते हैं।

राहुल देव बर्मन द्वारा रचित कुछ लोकप्रिय गीत

राहुल देव बर्मन ने हल्के फुल्के गीत भी बनाए और सुमधुर गीत भी। 'कच्ची कली कचनार की', 'लोगों की जुबान पर अपना नाम है पहला', 'गुलाबी आँखें जो तेरी देखीं', 'जय जय शिव शंकर कांटा लगे ना', 'ज़िन्दगी है खेल कोई पास कोई फेल', 'धीरे से जाना खटियन में है खटमल', 'महबूबा महबूबा', 'यम्मा-यम्मा यम्मा यम्मा', 'दम मारो दम', जैसे अनगिनत लोकप्रिय गीतों की उन्होंने रचना की, जिसके कारण वो आलोच्य भी रहे। इन सभी गीतों की तुलना में उन गीतों की संख्या अनगिनत हैं जिसके माध्यम से उन्होंने हिन्दी फ़िल्म संगीत जगत को एक नयी दिशा दी एवं आने वाले पीढ़ी के संगीतकारों के लिए मार्गदर्शक बने। उनके कुछ लोकप्रिय यादगार गीत हैं 'आजा पिया तोहे प्यार दूं', 'चिंगारी कोई भड़के', 'जिन्दगी है खेल कोई पास कोई फेल', 'दो नैनों में आँसू भरे हैं', 'हमें और जीने की चाहत न होती', 'क्या हुआ तेरा वादा', 'ज़िन्दगी के सफ़र में गुज़र जाते हैं', 'एक दिन मिट जाएगा माटी के मोल', 'छुकर मेरे मन को', 'सारा जमाना हसीनों का दीवाना' आदि। इन गीतों ने उनकी लोकप्रियता को बरकरार रखा। उनके बारे में यह कहा जाता है कि संगीतकारों की दुनिया में एक चर्चित और विवादित नाम था आर.डी. बर्मन। अपनी विशिष्ट 
संगीत रचना के माध्यम से वे हमेशा अपने आपको दूसरों से अलग साबित करने में सफल रहे। सही मायनों में पंचम की संगीत यात्रा सातवें दशक से प्रारम्भ हुआ। आठवें दशक तक आते-आते 'सनम तेरी कसम' (1981), 'जीवां (1986), 'जोशीले' (1989) इन सभी फ़िल्मों में दिया गया संगीत यह स्पष्ट करता है कि राहुल देव बर्म की जैसे-जैसे उमर बढ़ती जा रही थी, उनका संगीत अनुशासित यौवन का परिचय दे रहा था। 1985 तक राहुल देव बर्मन जी का स्वर्ण काल रहा। एक बार फिरसे 'परिंदा', 'मुस्कुराहट', 'द्रोही', 'गर्दिश' और 'गुरुदेव' जैसी फ़िल्मों में संगीत देकर राहुल देव बर्मन प्रचार में आ गए।

सन् 1961 में बनी फ़िल्म 'छोटे नवाब' से लेकर सन् 1996 में बनी फ़िल्म 'अन्याय ही अन्याय' इन तीन दशकों तक व्यापी आर.डी. बर्मन की संगीत यात्रा विद्वानों एवं गुणीजनों द्वारा सराही गयी है तथा उनका संगीत अत्यन्त लोकप्रिय भी रहा। फ़िल्म संगीत जगत से जुड़े गुणीजनों का यह मानना है कि राहुल देव बर्मन जी ने फ़िल्म संगीत को नई ऊँचाई दी। हिन्दी फ़िल्म संगीत जगत में पिछले दशकों में अनेक प्रतिभावान संगीतकार आये। परंतु सात के दशक के उत्तरार्द्ध से अगले दो दशक तक आर.डी. बर्मन की संगीत यात्रा धुआँधार चली। लोगों का यह मानना है कि राहुल देव बर्मन इस धारा में अंतिम मौलिक संगीतकार रहे। 'रॉक एण्ड रोल', 'डिस्को', 'ब्रेक' व 'रैप' इन सभी संगीतधाराओं का हिन्दी फ़िल्म संगीत में प्रयोग करके राहुल देव बर्मन ने अपनी मौलिक कार्य-कुशलता का परिचय दिया। 1984 में फ़िल्म 'इजाजत' के लिए उन्हें राष्ट्रीय पुरस्कार मिला एवं 1991 में उन्हें लता मंगेशकर सम्मान मिला। श्रेष्ठ संगीत निर्देशन के लिए उन्हें तीन बार फ़िल्म फेयर अवार्ड मिला - 'सनम तेरी कसम' (1982), 'मासूम' (1983) एवं '1942 ए लव स्टोरी' (1994) फ़िल्मों के लिए (उन्हें 1942 - ए लव स्टोरी के लिये अवार्ड मरणोपरान्त मिला)।

संगीत से सजी कुछ फ़िल्में

देशी पॉप संगीत के जम्मदाता राहुल देव बर्मन जी के संगीत से सजी कुछ यादगार फ़िल्में हैं 'प्यार का मौसम' (1961), 'छोटे नवाब' (1961), 'तीसरी मंजिल (1966), 'बहारों के सपने' (1967), 'पड़ोसन' (1968), 'कटी पतंग' (1970), 'अमर प्रेम' (1971), 'हरे रामा हरे कृष्ण' (1971), 'अपना देश', (1972), 'परिचय' (1972), 'जवानी दीवानी' (1972), 'सीता और गीता' (1972), 'मेरे जीवन साथी' (1972), 'अनामिका' (1973), 'यादों की बारात' (1973), 'नमक हराम' (1973), 'आपकी कसम' (1974), 'आँधी' (1975), 'शोले' (1975), 'महबूबा' (1976), 'बालिका वधु' (1976), 'किनारा' (1977), 'कर्मे वादें' (1978), 'नौकर' (1979), 'रॉकी तथा लव स्टोरी' (1980) 'शान' (1980), 'कालिया' (1981), 'याराना' (1981), 'सत्ते पे सत्ता' (1982), 'अंगूर' (1982), 'मासूम' (1983), 'सागर' (1985) इत्यादि। सन् 1980 के बाद फ़िल्म संगीत में एक नया दौर शुरू हुआ। उस समय उनकी सुरीली धुनों से बनी फ़िल्में थीं- 'कुदरत' (1981), 'सनम तेरी कसम' (1982), 'बेताब' (1983), 'मासूम' (1983), 'लव स्टोरी' (1981) 'इजाज़त' (1988) इत्यादि। सन् 1994 में बनी फ़िल्म '1942-ए-लव स्टोरी' में तो राहुल देव बर्मन जी का संगीत अति मधुर है।

पार्श्व गायक-गायिकाओं में राहुल देव बर्मन जी ने मन्ना डे, आशा भौंसले, मोहम्मद रफी, लता मंगेशकर, भूपेन्द्र, शैलेन्द्र सिंह, आरती मुखर्जी, सुरेश वाडकर, विनोद राठौड़, चित्रा, एस.पी. बालासुब्रह्मण्यम आदि सभी से गीत गवाएं, परंतु किशोर कुमार को उन्होंने अधिक महत्त्व दिया। नई पीढ़ी के तमाम गायक-गायिकाओं जैसे उदित नारायण, अमित कुमार, शब्बीर कुमार, कुमार शानू, 
कविता कृष्णमूर्ति, अल्का याज्ञनिक, इन सभी ने पहले पहले राहुल देव बर्मन जी के संगीत में ही गाया।

\section{उपसंहार}

'राहुल देव बर्मन जी एक ऐसे संगीत निर्देशक थे जिन्होंने युवा पीढ़ी को 'महबूबा-महबूबा', दो-प्रेमियों को 'मेरे सपनों की रानी कब आएगी तू' और उदास मन को भावपूर्ण गीत 'न कोई उमंग है न कोई तरंग है' जैसी सुरीली रचनाएँ सुनाई। उनके गीतों में एक सुरीला चुम्बक था जो श्रोताओं को खींच लेता था। हिन्दी फ़िल्म संगीत जगत को ऐसे ही एक संगीतकार का इंतजार है। हिन्दी फ़िल्म संगीत जगत में शुरू से लेकर अंत तक उनकी जो संगीत की यात्रा रही है उसके विषय में संगीतज्ञों का मानना है :

"लक्ष्मीकांत-प्यारेलाल की जोड़ी के प्यारेलाल शर्मा ने कहा : 'जो आर.डी. बर्मन की जीनियस के साथ न्याय कर सकें वे शब्द मैं कहाँ से लाऊं? वह तो उत्कृष्ट था। केवल वह था जो 'पड़ोसन', 'कटीपतंग' और 'महबूबा' जैसी परस्पर इतनी भिन्न प्रकार की तीन फ़िल्मों में, तीन प्रकार का संगीत दे सकता था। ... वह वाद्ययंत्रों के इस्तेमाल, रिकॉर्डिंग तकनीकों वगेरह के बारे में बहुविश्रुत (नौलजेबल) था। उनका संगीत भविष्य का संगीत है। जो उसने 30 साल पहले रचा वह अब रिमिक्स किया जा रहा है' ('बॉलीवुड मैलोडीज', 'गणेश अनंथरमन, पृ. 94)। पं. हरिप्रसाद चौरसिया के शब्दों में : वो आदमी तूफान था, भगवान का वरदान लेकर आया था। 'रसिकेषु' की 9 वीं कड़ी में अनिल बिस्वास के उद्गार ये थे: ... हमारे युग का आखिरी दिया जो बुझ गया है मगर उसके जैसा ऑलराउण्डर (सर्वगुणसंपन्न) न हुआ है कभी न होगा और यह मैं उसके पिता, स्वयं मुझे और सभी संगीत निर्देशकों को शामिल करते हुए कह रहा हूँ ... शाबाश बाप का शाबाश बेटा एक शब्द में कहें तो लाजवाब और उसके साथ हमारा जो भी है न वो खत्म हो जाता है। मेरे से उम्र में छोटा नहीं होता तो मैं उसे प्रणाम कर लेता... ${ }^{\prime \prime 1}$

4 जनवरी, 1994 को महान संगीतकार आर.डी. बर्मन नहीं रहे। उनकी याद संगीत प्रेमियों के दिलों में हमेशा रहेगी। अपने सदाबहार संगीत के माध्यम से वो सदा जीवित रहेंगे।

\section{संदर्भ ग्रंथ}

स्वरों की यात्रा, हिन्दी फ़िल्म गायकी का सफ़र (1931-2010), अनिल भार्गव।

स्वर-सरिता

पत्र-पत्रिकाएँ

अमर-उजाला।

जनसत्ता।

दैनिक भास्कर।

हिन्दुस्तान, नई दिल्ली।

नवभारत टाइम्स।

संगीत नाटक अकादमी, ग्रंथागार।

दिल्ली विश्वविद्यालय के संगीत एवं ललित कला संकाय के ग्रंथागार।

1 स्वर सरिता, हिन्दी फ़िल्म संगीत-विजय वर्मा-34, प्रकाशन तिथि, 1 सितंबर 2014, पृ. 37. 